\title{
Efficient supersonic air vehicle design using the Service-Oriented Computing Environment (SORCER)
}

\author{
Scott A. Burton ${ }^{1, *}$, Edward J. Alyanak ${ }^{2}$, and Raymond M. Kolonay ${ }^{2}$ \\ 1 American Optimization LLC, Springboro, Ohio 45066, USA \\ 2 Aerospace Systems Directorate Air Force Research Laboratory, Wright-Patterson Air Force Base, Ohio 45433, USA
}

Received 4 March 2014 / Accepted 18 December 2014

\begin{abstract}
The Air Force Research Lab's Multidisciplinary Science and Technology Center is investigating conceptual design processes and computing frameworks that could significantly impact the design of the next generation efficient supersonic air vehicle (ESAV). The ESAV conceptual design process must accommodate appropriate fidelity multidisciplinary engineering analyses (MDAs) to assess the impact of new air vehicle technologies. These analyses may be coupled and computationally expensive, posing a challenge due to the large number of air vehicle configurations analyzed during conceptual design. In light of these observations, a design process using the Service-Oriented Computing Environment (SORCER) software is implemented to combine propulsion, structures, aerodynamics, aeroelasticity, and performance in an integrated MDA. The SORCER software provides the automation and tight integration to grid computing resources necessary to achieve the volume of appropriate fidelity analyses required. Two design studies are performed using a gradient-based optimization method to produce long and short range ESAV wing designs. The studies demonstrate the capability of the ESAV MDA, the optimization algorithm, and the computational scalability and reliability of the SORCER software.
\end{abstract}

Key words: Optimization, Air vehicle design, SORCER.

\section{Introduction}

The ability of a new aircraft design to meet various requirements is largely dictated by decisions made during the conceptual design phase $[1,2]$. Specifically, it is the conceptual design that determines the majority of the aircraft's life cycle costs [3]. It is therefore advantageous to assess as many air vehicle designs with the highest fidelity analyses possible given the available resources (e.g., human, schedule, and computational) This tradeoff between number of designs analyzed and available resources has typically favored the use of low fidelity closed-form equations and statistical models in conceptual design. These computationally inexpensive tools facilitate the assessment of large numbers of designs, but typically lack the fidelity to uncover significant engineering risks or are unable to assess the impact of new technologies.

In recent years, advances in computer processing speed and the prevalence of grid computing resources in industry have opened the door to more computationally expensive analyses during conceptual design [1, 4]. The conceptual design process described herein is based on the availability of such resources and incorporates analyses from several engineering

*e-mail: scottaburton@amopti.com disciplines. The efficient supersonic air vehicle (ESAV) multidisciplinary analysis (MDA) is used in a design example that combines techniques from traditional conceptual and preliminary design processes. Details of the ESAV MDA are discussed in Section 2.

While the existence of grid computing facilities is a necessary condition for performing large-scale conceptual design studies, it is not sufficient. Software to integrate the different engineering analysis codes and coordinate the execution of thousands of analyses remains an area of research. The study herein uses the Service-Oriented Computing Environment (SORCER) software to perform these functions [5]. Salient features of the SORCER ESAV application are discussed in Section 3. The ESAV MDA is exercised on two optimization problems described in Section 4. Lastly, results of the ESAV design studies and conclusions are presented in Sections 5 and 6 , respectively.

\section{Multidisciplinary analysis}

The MDA described herein is a blend of conceptual and preliminary analysis methods from geometry, propulsion, structures, aerodynamics, aeroelasticity, and performance 


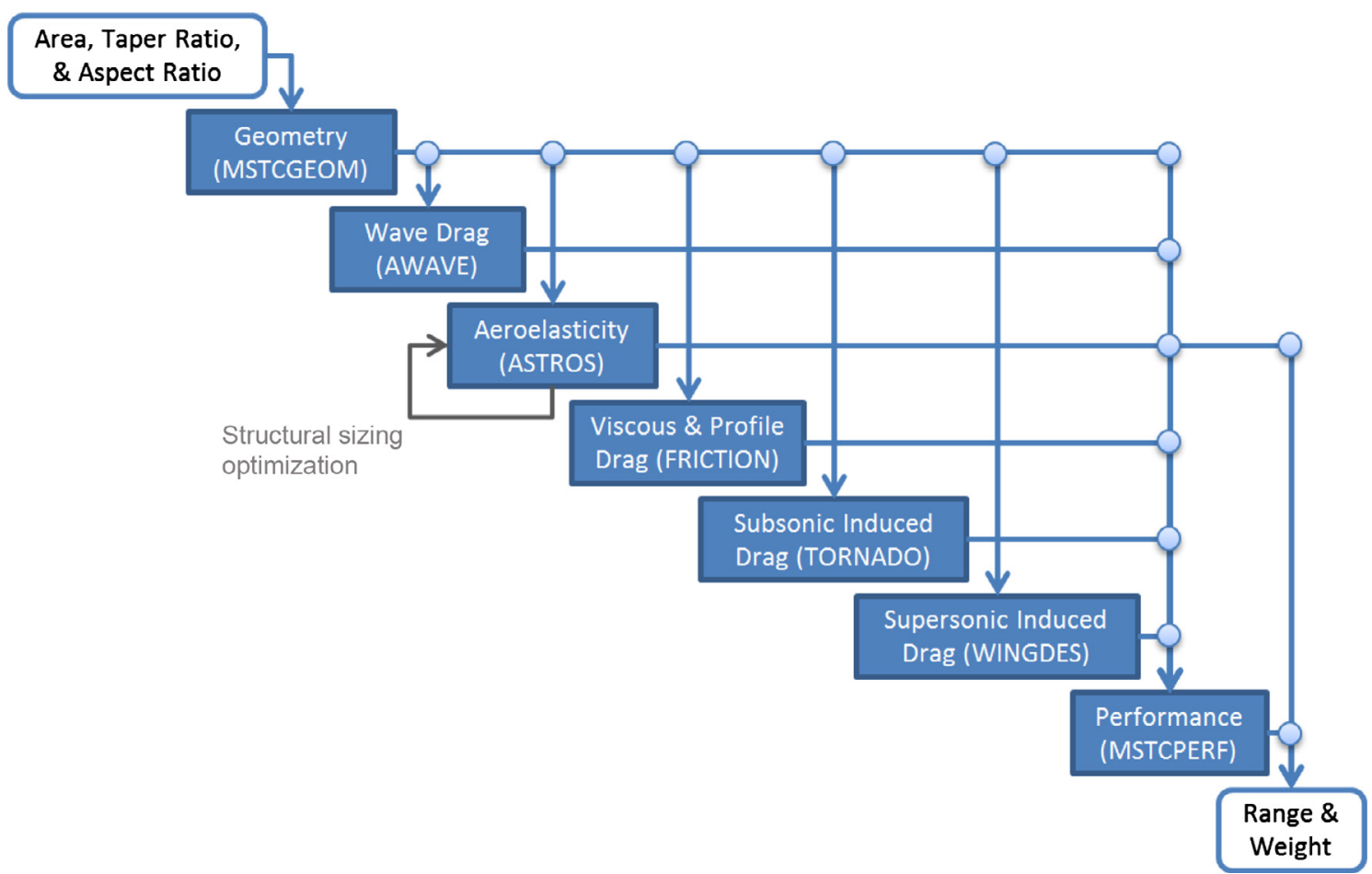

Figure 1. The ESAV MDA $N^{2}$ diagram includes geometry generation, an aerodynamic analysis, an aeroelastic analysis with structural sizing optimization, and a performance analysis.

disciplines. The analysis process and data flow is shown in the ESAV $\mathrm{N}^{2}$ diagram in Figure 1.

The process begins by parametrically generating geometry, mesh, and input data suitable for several different analyses at varying fidelities. The geometry is used as input to compute several figures of merit of the air vehicle, which include the air vehicle drag polars, design mass, range, and aeroelastic performance. The different responses are evaluated for several flight conditions and maneuvers. These responses are then used to construct the objective and constraints of the multidisciplinary optimization (MDO) problem.

\subsection{Airframe geometry}

The purpose of the airframe geometry application, MSTCGEOM, is to create geometry data for the physics-based analyses in the MDA. MSTCGEOM is compiled Matlab ${ }^{\mathrm{TM}}$ code and is considered medium fidelity [6]. The application does not replace or replicate the extensive functionality of a commercial CAD package (i.e., a high fidelity geometry application); however, it does go beyond the functionality of a typical conceptual design tool. MSTCGEOM parametrically creates mid-plane panel aerodynamic models and FEM analysis and design models. In the case of ASTROS or NASTRAN, these FEM models may include static and dynamic aeroelastic load cases. In addition, composite structural elements and distributed masses to represent sub-systems may be a part of the FEM models. The geometric features of the aircraft modeled include the fuselage and all of the lifting aircraft components (wings, vertical stabilizer, and horizontal stabilizers). The physics-based analyses that use the MSTCGEOM output include ASTROS, FRICTION, TORNADO, AWAVE, WINGDES, and MSTCPERF [7-9].

MSTCGEOM is computationally inexpensive to execute and produces the required fidelity output for the ESAV analyses described herein. In this manner, MSTCGEOM is a bridge between conceptual and preliminary design fidelities. MSTCGEOM may be used to make both parametric and topological changes to the airframe wing during the MDO. Figure 2 shows an example of the MSTCGEOM application output for the baseline ESAV.

MSTCGEOM works by building up a series of major components such as cross sections, wing sections, point masses, and material definitions into assemblies such as wing components and a fuselage. Each of these components and assemblies are objects that contain methods that return information to support analysis model generation for a specific fidelity or type of model. For example, a cross section object may return midplane information to support the generation of a linear aerodynamic panel model. Likewise, the same cross section object can also return a completely discretized representation of itself for use in a FEM (i.e., the logic to produce both models exists in the same object). Any aerodynamic or structural mesh that is produced may be refined based on the user's requirements. The level of refinement used in the ESAV analyses is accurate enough to produce the correct physics-based global trends, but is not sufficient to identify stress concentrations that may be used in fatigue life prediction. 

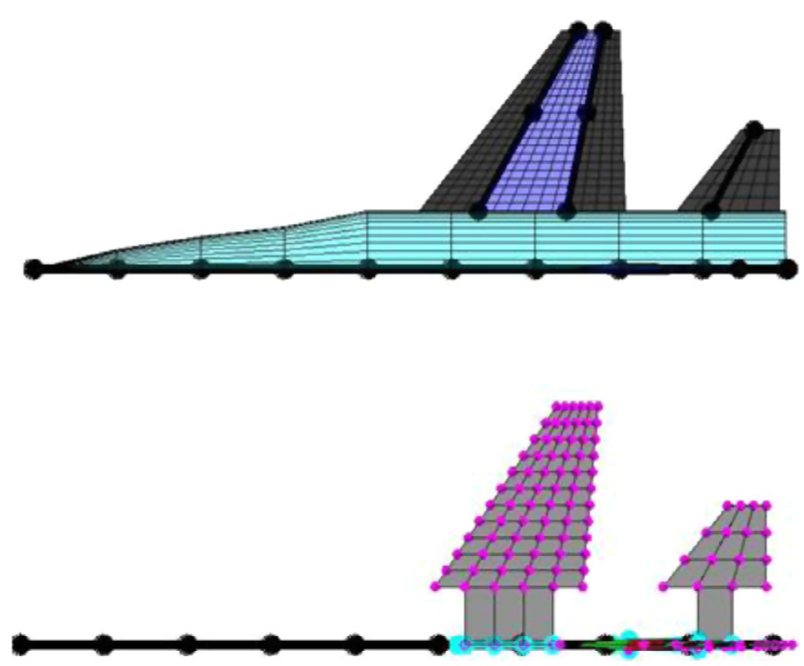

Figure 2. The MSTCGEOM application creates discrete geometry for ASTROS, FRICTION, TORNADO, AWAVE, WINGDES, and MSTCPERF applications (baseline ESAV half-span shown).

\subsection{Aeroelasticity}

Stress analyses and aeroelastic sizing for the ESAV are performed for four static aeroelastic maneuver cases at an altitude of 10,000 ft. Specifically, they are the following: (1) a $9.0 \mathrm{~g}$ pull-up at Mach 0.9; (2) a $7.2 \mathrm{~g}$ pull-up at Mach 1.2; (3) a negative $3.0 \mathrm{~g}$ push-over at Mach 1.2; and (3) an anti-symmetric $100 \mathrm{deg} \mathrm{s}^{-1}$ aileron roll at Mach 1.2. ASTROS is used to perform the aeroelastic analysis and structural sizing optimization that results in an optimized structural weight subject to stress constraints. The structural sizing optimization is considered a sub-optimization in regards to the overall optimization problem discussed in Section 4.1. To that end, the structural sizing optimization is an integral part of the MDA and is performed for each configuration analyzed. The structural sizing suboptimization problem has 57 design variables that are distributed over the wing and tail surface spar, rib, and skin thicknesses. The ESAV is modeled with aluminum and $70 \mathrm{ksi}$ is used as the maximum allowable stress.

\subsection{Aerodynamics}

To assess the aero performance of a configuration, estimations of zero-lift drag and induced drag are required. The zero lift drag components estimated are viscous and profile drag. They are calculated using the FRICTION code [8]. Solutions for zero lift drag are computed at a number of altitude and Mach numbers. Specifically, the altitudes are: $0 ; 10,000$; 30,000 and $60,000 \mathrm{ft}$. The Mach numbers are: $0.0 ; 0.1 ; 0.5$; $0.9 ; 1.0 ; 1.1 ; 1.5$; and 2.0 .

The induced drag is estimated using the vortex lattice code TORNADO [9]. A TORNADO solution is computed over a range of angles of attack and Mach numbers and combined with the FRICTION results to develop a set of drag polars. The resulting set of drag polars are used in the mission performance calculations for each configuration.
For the structural sizing problem described in the previous section, the aerodynamic analysis is internal to the ASTROS application. Specifically, USSAERO is the aerodynamic solver used in the version of ASTROS for the study herein. The USSAERO aerodynamic model contains all the lifting and control surfaces.

\subsection{Propulsion}

The aircraft engine performance is modeled using TERMAP (Turbine Engine Reverse Modeling Aid Program). TERMAP is an engine cycle performance program developed by Allison Engineering that uses a building block approach to predict both the steady state and transient performance of gas turbine engines [10]. The program is FORTRAN-based and requires a user to define engine component characteristics. TERMAP uses this information to estimate flow path information at station numbers throughout the engine.

To improve the computational efficiency of the $\mathrm{N}^{2}$ process in Figure 1, TERMAP is used to build an engine deck approximation of thrust and specific fuel consumption over all flight conditions of interest. The engine deck is used during the optimization to calculate range and optimal altitude trajectory for the flight leg of interest.

\subsection{Mission performance}

The mission performance assessment involves calculating the aircraft range for a given Mach number. This is accomplished by using the design weight from ASTROS. This FEM-based weight calculation is higher fidelity than an empirically-based calculation typically done in conceptual design. The design weight includes non-structural masses and internal fuel load, such that the design weight is the gross takeoff weight for the vehicle. (Since a half-span model is used, the weight from ASTROS is half the takeoff gross weight.) The design weight along with the drag polars and the propulsion system performance assessment are used to calculate the range for the vehicle.

The range calculation assumes that the air vehicle is flying at an optimal altitude for its weight. The altitude is allowed to increase as fuel is burned, thus returning a range equal to the distance traveled over the optimal altitude trajectory. For the ESAV studies herein, the range calculation is done for a $6000 \mathrm{lb}$ fuel burn at Mach 0.8.

\section{Multidisciplinary analysis implementation}

The practical large-scale MDO of an ESAV requires the MDA behind the objective and constraint functions be implemented such that the following hold true: (1) the evaluation of constraints and objectives is automatic and does not require user intervention; (2) the evaluation of constraints and objectives is reliable, repeatable, and accurate to the desired fidelity; (3) constraint and objective evaluations that encounter errors or exceptions are communicated to the MDO algorithm gracefully and the MDO algorithm handles them appropriately; 
and (4) the evaluation of objective and constraint functions for different designs is done in parallel using the computational resources available.

With these considerations in mind, the Java-based ServiceOriented Computing Environment (SORCER) software is used to implement the ESAV MDA [5]. The MDA is then driven by an optimization algorithm implemented in Matlab via the SORCER ModelClient class.

\subsection{SORCER fundamentals}

SORCER is a Java-based, network-centric computing platform that enables engineers to perform analyses and design studies in a very flexible, robust, and distributed computing environment. At the foundation of an engineering analysis within SORCER is the concept of a service provider. A service provider - or simply "provider" - is Java code implemented in accordance with SORCER standards that makes a number of Java methods available to remote clients over a computer network [11]. These methods are referred to as the provider's service operations.

Providers typically provide service operations that leverage existing domain-specific analysis codes (e.g., CFD or FEM codes). These providers are referred to as analysis providers. The implementation of a given service operation in an analysis provider usually involves wrapping an engineering analysis executable with Java code. The executable is generally platform dependent and performs the bulk of the engineeringspecific computations for a given service operation. An analysis provider's service operation may also enhance the functionality of the underlying executable with Java code or simply pass data to-and-from it.

Two common approaches to wrapping executables are to: (1) use the file system in conjunction with a system call from the analysis provider's service operation; or (2) use more sophisticated technology such as Java Native Interface to tightly couple the analysis provider's service operation to the executable. In the first case, the service operation writes an input file for the executable to read, invokes the executable via a system call, and concludes by reading the output file produced by the executable. The service operation may return the raw output files produced by the executable via a URL object or post-process the file and pass higher-level Java objects to the remote client. If errors or exceptions occur in an analysis provider's service operation, the caller is notified gracefully and may (or may not) take action to correct the problem. Once an analysis provider has been implemented to provide a number of service operations, it may be published from a computer on the network using SORCER. Remote clients may then access the analysis provider's service operations via a small Java program called a service requester, or use simple SORCER command-line tools to access service operations directly.

For example, the service operation used in the ESAV study to create input files for ASTROS is called doMstcGeomService. This service operation is implemented by a provider called Engineering Air Vehicle Provider. The underlying executable for the doMstcGeomService is the compiled Matlab program MSTCGEOM. MSTCGEOM creates all of the input decks required for the individual analysis tools in the ESAV MDA. The argument for the Java method doMstcGeomService is an object that contains all the information necessary to define the MSTCGEOM input deck, including the ESAV wing design variables (area, aspect ratio, and taper ratio). Using the input object, the Engineering Air Vehicle Provider's doMstcGeomService service operation writes an input deck for the MSTCGEOM Matlab executable, invokes MSTCGEOM via system call, and returns the URL of the ASTROS input file produced by MSTCGEOM to the remote client. In addition to the ASTROS input file, the MSTCGEOM executable also writes input files required by the doTornadoService and executeFriction service operations. Both of these service operations are also provided by the Engineering Air Vehicle Provider.

Like most service operations, the doMstcGeomService service operation returns multiple outputs that may be used by multiple downstream service operations to form a complete MDA. The returned objects from a service operation may be used directly or post-processed on the client-side (i.e., at the user's location) such that the output may be meaningful for design optimization. In the previous example, the output URL object pointing to the ASTROS input file is used directly by a downstream service operation. If a scalar-value was desired - e.g., to assign a value to an objective function during an optimization - the URL could be opened and parsed to yield a number. The means of reducing output from service operations in this manner is accomplished with the SORCER Filter subclasses, discussed later.

To provide a layer of abstraction from a specific implementation of a service operation (i.e., Java method), providers implement Java interfaces to identify the service operations they provide. (Java interfaces are defined by a list of method signatures, which are composed of a method name, argument types, and return type.) These Java interfaces are referred to as service types. In this manner, a remote-client is generally only concerned about finding a provider that implements the service type containing the service operation of interest. The specific provider usually does not matter, since it is the service operation that the client desires. Many providers on the network may have different implementations of the same service operation in which case they would all implement the same interface (i.e., service type). If a client desires to select a specific provider for a given service operation, the client may specify the name of the provider in addition to the service type.

To use SORCER providers that are published on a network, a client must specify the name of the service operation, the service type that contains the service operation of interest, and the arguments for the service operation. The client does not have to specify the hostname, port, or IP address of the computer that is running the service operation process. The underlying architecture, Jini Connection Technology, supports dynamic discovery and join on both the provider- and client-side, thus relieving the client from knowing where to find service operations.

The arguments for all SORCER service operations are instances of the Context class. A Context object - or "context" 


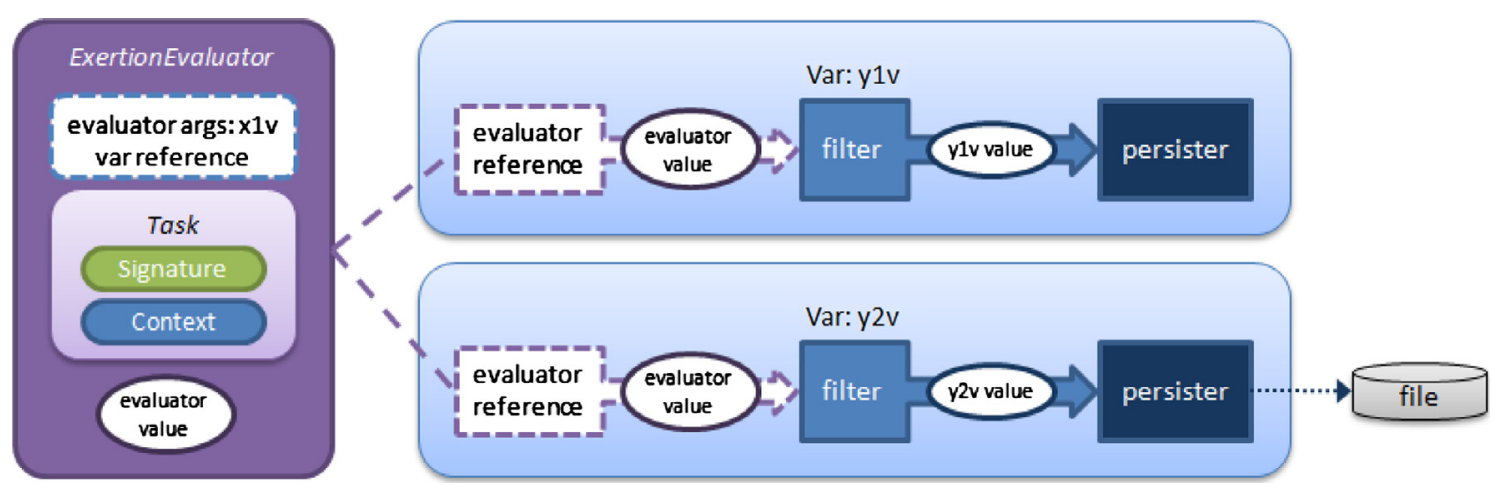

Figure 3. A single Evaluator object may be referenced by multiple Var objects employing different filters to reduce aggregate data to scalar values; the values may be then written to a file (or object field) using a var's persister.

- is a generic container that encapsulates name-value pairs. In this manner, a context possesses its own namespace for identifying argument objects for a service operation. The service operation name and service type are encapsulated in the SORCER ServiceSignature class. (Instances of ServiceSignature are referred to as "signatures".)

The combination of the service operation name, service type, and a context are encapsulated in an instance of the SORCER Task class. A Task instance - or "task" - represents the basic unit of work that service providers operate on. Since tasks involve using remote service operations, a layer of abstraction exists to encapsulate both remote and locally executed units of work. The Evaluator class and its subclasses are the entities responsible for doing computational work via their evaluate method from both remote and local resources in SORCER.

An Evaluator instance - or "evaluator" - that encapsulates a task is called an exertion evaluator. The word "exertion" implies the remote use of service providers. This is in contrast to other subclasses of Evaluator that are capable of doing simple calculations locally when the heavy lifting nature of an engineering analysis service operation is not required. For example, the ExpressionEvaluator class enables a client to combine Var instances in algebraic equations without using a remote service operation (the Var class is discussed later). In this light, evaluators are the entities responsible for either defining the process of how data is produced via remote service operations or producing the data themselves locally via their own logic.

Var instances - or "vars" - are used to form both independent and dependent variables in SORCER. In the case of independent variables, vars have an instance of IndependentEvaluator that contains the value of the independent variable. The IndependentEvaluator object acts as a simple container to store a value and does not perform any calculations or use service operations on the network.

Dependent vars are typically created to implement scalarvalued mathematical functions, such as objective, constraint, or other mathematical functions for use in analysis or optimization studies. They may also be used to form composite functions. Vars contain the objects necessary to produce - rather than store - the values they represent, which may include Evaluator, Filter, and Persister instances. The three types of objects are used in a specific sequence when the method getValue is invoked on a dependent var object.

When the getValue method is called on a dependent var, the evaluator's getValue method is subsequently called. The evaluator's getValue method then, in turn, calls getValue on its argument vars to ensure their respective values are current before proceeding. (If a var represents a function dependent on other vars, i.e., argument vars, the evaluator of the var representing the function holds references to those argument vars.) The evaluator then checks to see if it is out of date due to argument vars changing since the last invocation of evaluate. If the argument vars have not changed, the evaluator returns its current value without further processing. In this manner, all calls to evaluate are demand-driven, i.e., the evaluate method is invoked only if the evaluator's arguments have changed since the last invocation of evaluate. As described earlier, the evaluate method may use a remote service operation implemented by a provider on the network to perform the necessary calculations, or the calculations may be done locally via its own logic. Once the evaluator's evaluate and getValue methods complete and return a new value to the var, an instance of a Filter subclass may be employed.

Since exertion evaluators typically return aggregate forms of data, reducing the output from them is often necessary. This data reduction is accomplished using the Filter subclasses. Using a Filter subclass object - or "filter" - on the returned objects from an evaluator allows clients to reduce or transform the aggregate data produced by evaluators to yield new objects. The result of filtering is often a scalar value of a mathematical function. In the case where an evaluator produces aggregate data, the evaluator may be referenced by a number of different vars with different filters to yield different values, see Figure 3. A series of filters may be combined to form a filter pipeline, such that downstream filters operate on the output of upstream filters. The resulting value returning from the filter (or pipeline filter) is the value of the var, which is then be passed to the var's Persister instance if it exists.

The Persister instance - or "persister" - uses the value from the filter as an argument to its setValue method. Depending on the specific subclass of Persister used, the persist method may write the var value to a file or set a field on an object. Once the persister is done executing, the var value is returned to the caller of the var's getValue method. 


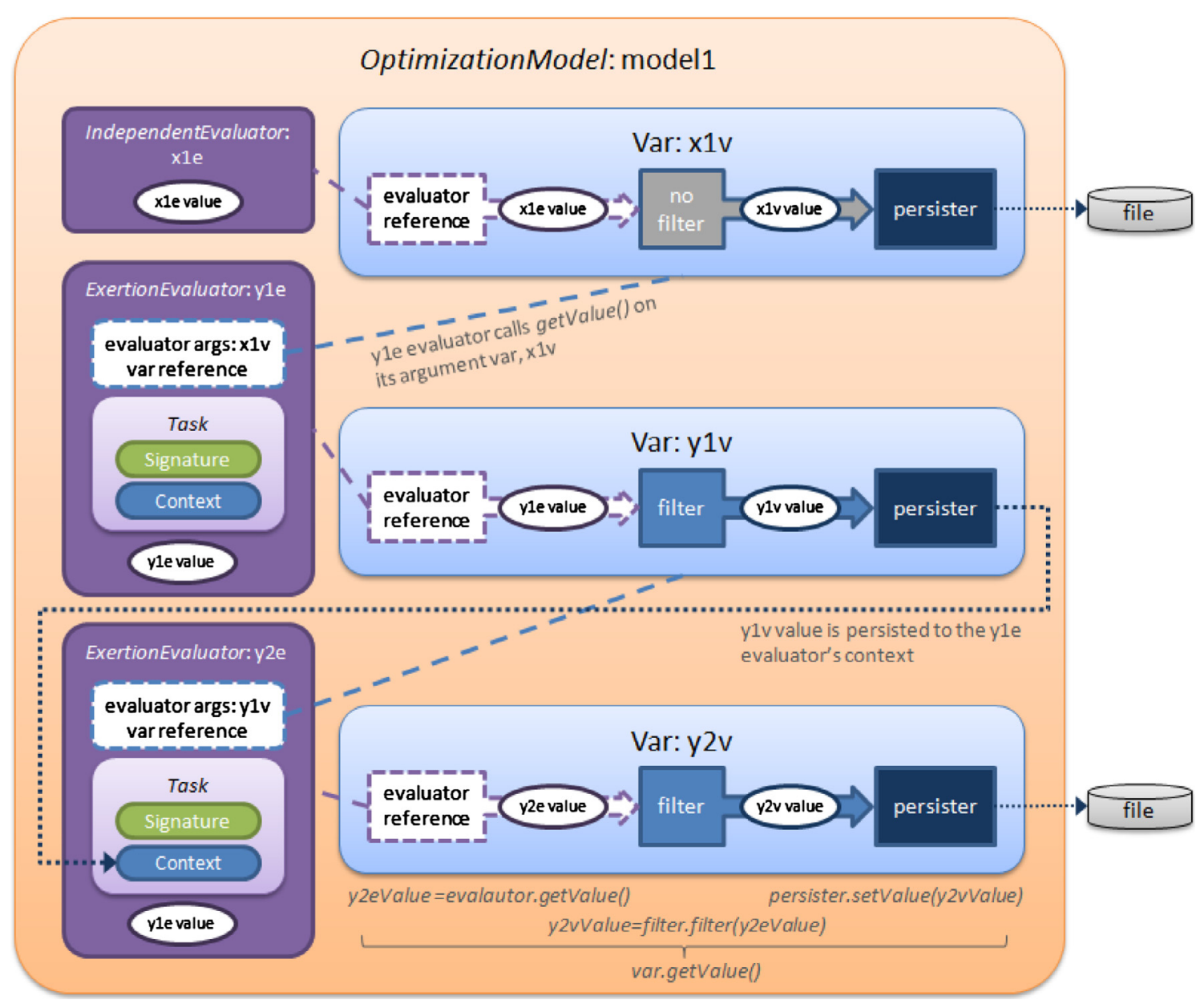

Figure 4. An OptimizationModel is a collection of Var objects; each var references an evaluator and may be referenced by other vars to form composite functions.

An OptimizationModel instance - or "model" - is a collection of any number of vars that define a specific optimization problem. Figure 4 illustrates a simple example of an OptimizationModel called modell. The model contains three vars; one independent and two dependent. The var $y l v$ is a function of $x l v$, which is the independent var. The var $y 2 v$ is a function of $y 1 v$, thus it is a composite function.

The vars are the implementation of the design variables, optimization objective function, and constraint functions. Additional vars may be included for monitoring other figures-of-merit. Once a model is created, it may be published as a provider for clients to access remotely. This type of provider is referred to as a model provider to distinguish it from analysis providers. The model provider behaves like shared memory that is accessible to remote clients over the network. There is only one state of the model, which is subject to manipulation by remote clients. There are two techniques for clients to interact with a published model provider: (1) via a single model query; or (2) via a table model query.

A single model query is done from a requestor or SORCER command-line tool. In both cases, a query object is constructed containing the design variable var names and values and the var names of the objective and constraint functions that the client wishes to calculate. The query also contains the name of the model provider, so that it may be found on the network.

In contrast to analysis providers, accessing model providers over the network does not require clients to specify a service type and service operation name. Since all OptimizationModel instances have only one service type with a single service operation, the information is implied. This does, however, necessitate that the client specify the model provider name when multiple models are published.

Once the query is constructed, it is executed by the client. The published model provider receives the query object and proceeds to invoke setValue on all the design variables. Once the setValue calls are completed, the model begins calling getValue on the client-specified objective and constraint functions. After all the getValue calls are complete, the query object is returned to the remote client with the updated var values.

The second technique of using models is via a table query. Rather than passing a single set of design var values to the model provider, a client may construct a table of runs containing the names of the design vars and their values for each run. The table is added to the query with the name of the model provider and the var names of the objective and constraint vars the client wishes to calculate. Since a table of runs is being sent 


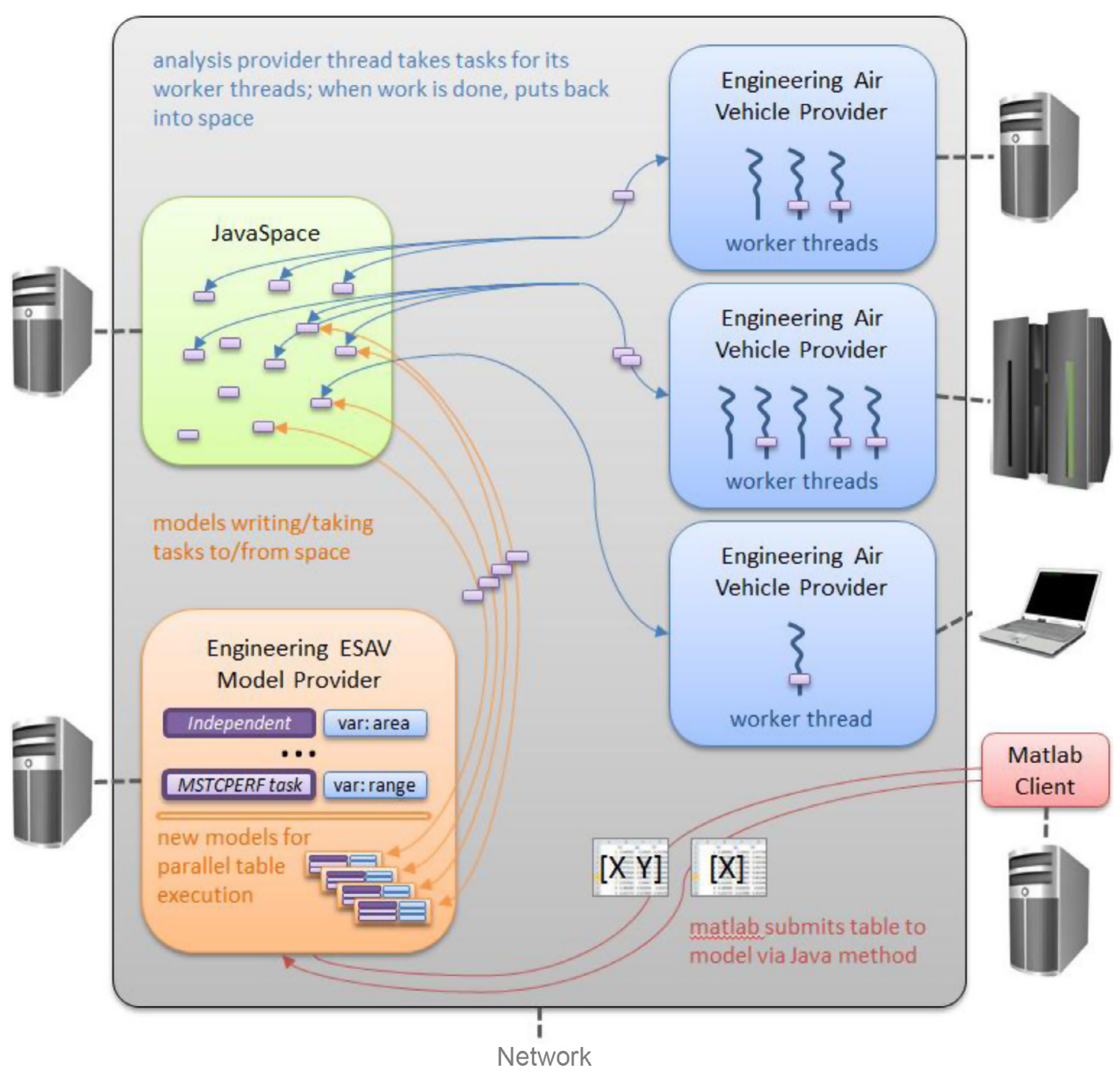

Figure 5. SORCER uses JavaSpaces technology to provide a flexible, dynamic grid computing facility for ESAV optimization studies.

to the model provider, the client may specify the number of runs the model provider is to execute in parallel.

Once the table query is constructed and sent to the model provider, the model provider creates new child instances of itself for parallel execution of the table. (The child instances are newly constructed objects, such that the states of their respective vars are not inherited from the model provider.) The model provider then creates a thread for each child model and begins to setValue and getValue on the vars as in the single model query. Once all the threads complete, the var values for each run are returned to the caller in a table object.

In contrast to the single model query, the table query does not affect the state of the parent model provider's vars. The child models are isolated from one another and the parent model, and are used exclusively to perform the client-requested calculations. Once the calculations are complete, the child models are discarded and the states of the parent model vars remain unaffected.

\subsection{SORCER space computing}

Large numbers of engineering analyses are generally required to perform conceptual-level aircraft design. This significant computational burden is addressed at the AFRL MSTC by using the SORCER software. The network-centric approach of SORCER enables the use of heterogeneous computing resources, including a variety of operating systems, hardware, and software. Specifically, ESAV studies performed at the AFRL MSTC use SORCER in conjunction with a mix of Linux-based cluster computers, desktop Linux-based PCs, Windows PCs, and Macintosh PCs. The ability of SORCER to leverage these resources is significant to MDO applications in two ways: (1) it supports platform-specific executables that may be required by an MDA; and (2) it enables a variety of computing resources to be used as one entity (including stand-alone PCs, computing clusters, and high-performance computing facilities). The main requirements for using a 

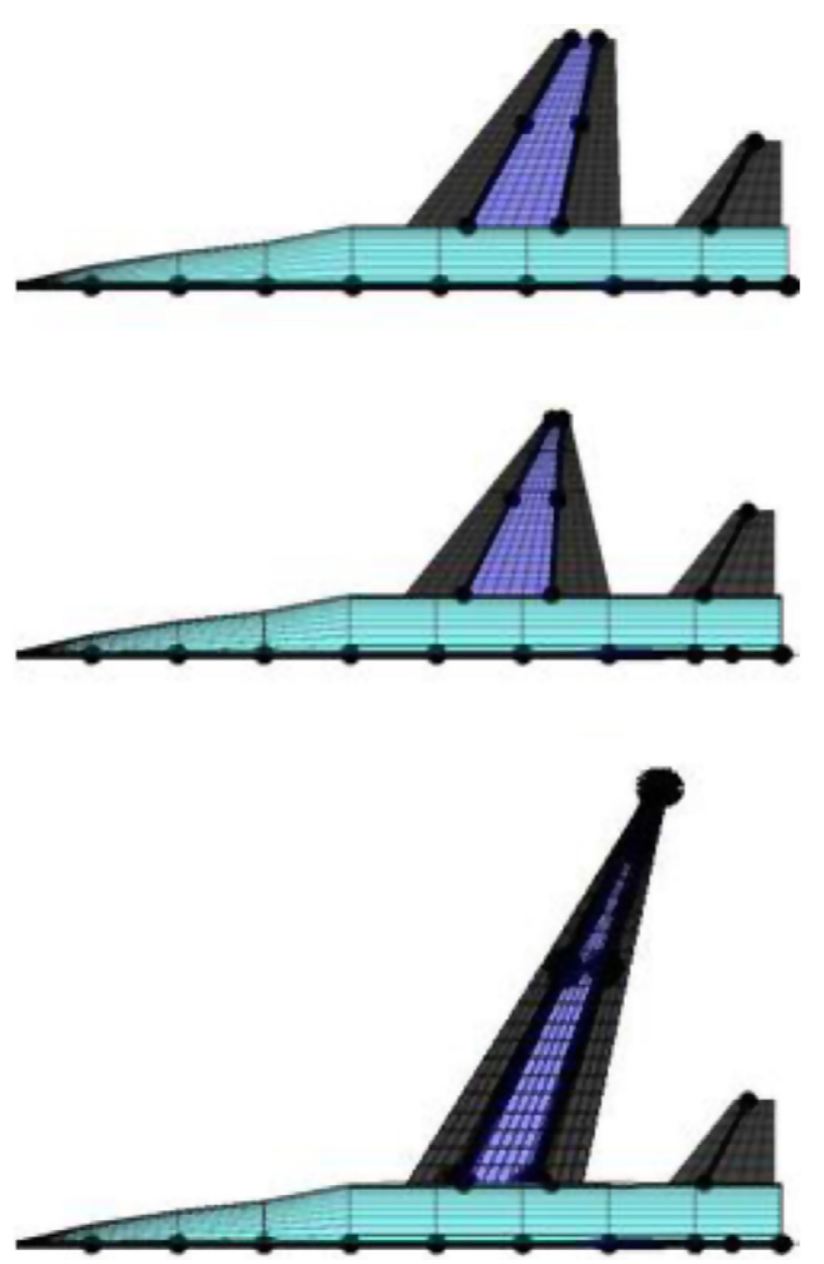

Figure 6. The ESAV optimization result half-span planforms: baseline $1550 \mathrm{mi}$ range and 13,125 $\mathrm{lb}$ weight (top); $1500 \mathrm{mi}$ range and 13,013 lb weight (middle); and $2500 \mathrm{mi}$ range and 14,065 lb weight (bottom).

computational resource in SORCER are network connectivity and Java compatibility. SORCER also supports load balancing across computational resources via the JavaSpaces technology, making the evaluation of objective and constraint functions in parallel a simple and a dynamically scalable process [12].

SORCER employs JavaSpaces technology to implement a loosely-coupled distributed computing system. JavaSpaces enables different processes on different computers to communicate asynchronously in a reliable manner [12]. Using this technology, SORCER implements a self-load balancing grid computing system that can dynamically grow and shrink during the course of an optimization study. The self-load balancing quality of SORCER is necessary to ensure efficient use of computational resources given the varying computational expense of different tasks executing simultaneously during a design study [13].

A JavaSpace - or "space" - is Java code running on a computer that is accessible on the network in the same manner as service providers, see Figure 5. The space provides a type of shared memory where exertion evaluators can put tasks they wish to be processed by service providers. Service providers, in turn, use Jini discovery mechanisms upon startup to find spaces on the network and monitor them for tasks with their service type. If a service provider sees a task it can operate on in a space, and the task has a flag indicating it has not been processed, the provider takes the task from the space. The provider then executes the appropriate service operation and returns the task to the space with a flag indicating the task has been processed. Once the task has been returned to the space, the process that initially wrote the task to the space detects the returned task and checks to see if it has been processed. If the task indicates it has been processed, the evaluator removes the task from the space.

To achieve the load balancing across multiple computers, a service provider may be configured to have a fixed number of worker threads. The number of worker threads determines the number of tasks a provider can process in parallel. By configuring the number of worker threads for a specific service provider on a specific computer, the provider can self-load balance the computer it is hosted on (assuming that it is the only service provider operating on the host computer).

\section{Design studies}

The design of a supersonic air vehicle involves assessing many figures of merit and the tradeoffs between them. In this study, a preliminary exploration of the design space is performed through univariate parametric sweeps of the design variables. In this manner, each design variable is independently swept from its lower bound to its upper bound in equal steps. This process is costly, but it serves three important purposes: (1) the sweeps provide a set of results that engineers may use to assess the validity of the MDA implementation; (2) the results may be used to screen design variables and responses for significance, which may result in a smaller MDO problem; and (3) the sweep results are used to calculate the sensitivities for the first iteration of the gradient-based optimization process. Once the preliminary study is completed, the MDO problem is solved using a robust successive linear programming (SLP) technique.

\subsection{Optimization problem statement}

A single objective function is identified along with nonlinear and side constraint functions to exercise the ESAV model in SORCER. The optimization problem takes the general form:

$$
\left\{\begin{array}{c}
\text { Minimize } f(\boldsymbol{x}) \\
\text { subject to } \boldsymbol{g}(\boldsymbol{x}) \leq \mathbf{0} \\
\text { and } \boldsymbol{x}^{\mathrm{lb}} \leq \boldsymbol{x} \leq \boldsymbol{x}^{\mathrm{ub}} \\
\text { where } \boldsymbol{g} \in \mathbb{R}^{n c o n} \text { and } \boldsymbol{x} \in \mathbb{R}^{n d v} .
\end{array}\right.
$$

The objective is to minimize the structural weight of the ESAV, which includes the fuselage and lifting surface weights. In this manner, the life cycle cost of the aircraft is kept down due to its correlation with takeoff gross weight [14]. Each evaluation of the objective function, $f(\boldsymbol{x})$, involves a structural sizing sub-optimization of the wing and tail surface spar, rib, and 

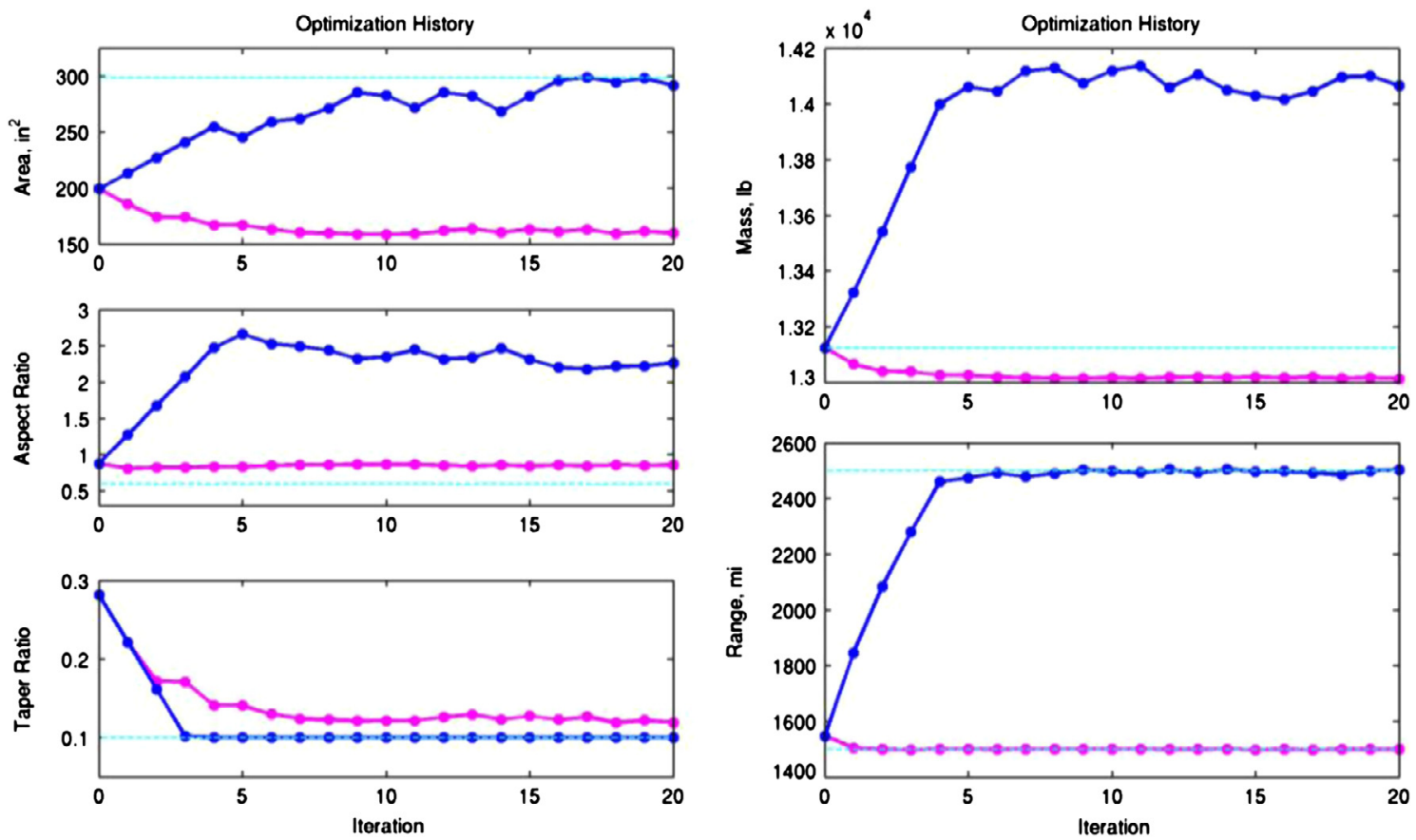

Figure 7. Results from ESAV optimization illustrate how the design variables, objective and constraint functions are manipulated by the SLP technique (1500 mi range constraint, magenta; $2500 \mathrm{mi}$ range constraint, blue).
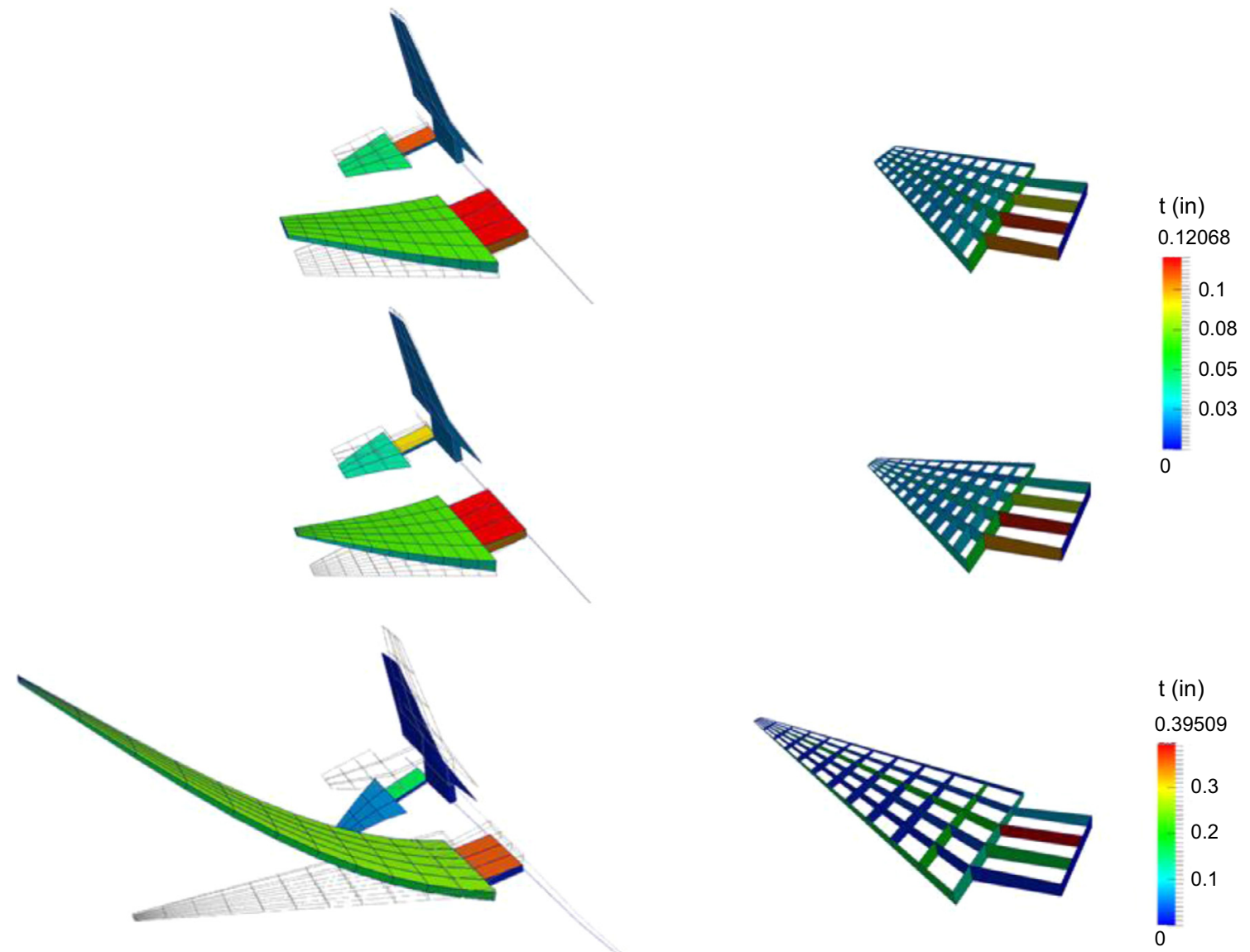

Figure 8. The ESAV planform and structural design thicknesses from the ASTROS structural sizing sub-optimization problem are shown above for the $1550 \mathrm{mi}$ range baseline design (top row), the $1500 \mathrm{mi}$ range design (middle row), and the $2500 \mathrm{mi}$ range design (bottom row) optimal designs. (The left column shows the thicknesses for the wing skins and the right column shows the thicknesses of the ribs and spars.) 
skin thicknesses. This sub-optimization process is performed by ASTROS and is integral to the MDA, see Section 2.2 for details.

Two optimization problems are solved with a single range constraint (ncon equal to one). In the first case, the cruise leg range, $g(\boldsymbol{x})$, is constrained to be above $1500 \mathrm{mi}$ at Mach 0.8 for $6000 \mathrm{lb}$ of fuel. In the second case, the range is increased to $2500 \mathrm{mi}$. The design variables, $\boldsymbol{x}$, are the wing area, taper ratio, and aspect ratio ( $n d v$ equal to three). The lower and upper bounds on the design variables $\left(x^{\mathrm{bb}}\right.$ and $\left.\boldsymbol{x}^{\mathrm{ub}}\right)$ are: 100 and $300 \mathrm{ft}^{2}$ for wing area, 0.6 and 5.0 for aspect ratio, and 0.1 and 1.0 for taper ratio, respectively.

\subsection{Optimization algorithm}

The optimization technique applied is an adaptation of the Frank-Wolf implementation of the successive linear programming (SLP) method [15]. It is a gradient-based method that uses a finite-difference approach to compute sensitivities. Issues of computational efficiency and robustness are addressed using parallel computations with SORCER and high-order/adaptive finite-differencing techniques.

The SLP method used herein is tailored for parallel computing on a large number of CPU cores such that gradient and line search calculations are executed in parallel [16]. The algorithm is tolerant to failed runs in both the gradient and line search phases of an optimization iteration. This robustness comes with the computational cost associated from the extra analyses required over that of a typical first-order finite-difference technique.

To ensure a sensitivity calculation is successful, four objective and constraint function evaluations are executed in parallel for each design variable at each SLP iteration. (The number of points used in the sensitivity calculation is based on the computational resources available and the experience of the designer.) The objective and constraint evaluations are used to calculate the highest-order finite-difference sensitivity possible given the number of successful function calls. If all of the function calls fail, the robust SLP algorithm attempts to adapt the step-size and recalculate the sensitivity. If this attempt fails, the user is prompted to take corrective action.

The optimization begins by calculating the gradient of the objective and constraint functions at the initial design point. The gradients are then used to form a constrained linear programming (LP) problem where the optimum is found using an LP solver. In traditional implementations of gradient-based methods, a line search follows that involves sequentially searching for a minimum along a line from the initial design to the estimated optimum. This approach is inefficient given the computing resources available to most engineers today.

Since many CPU cores are available on a single computer, cluster, or high-performance computing facility, the line search is converted to a partial enumeration of the line. The number of designs evaluated on the line depends on the computational resources available, the anticipated nonlinearity of the search, the computational expense of the analysis, and the schedule of the MDO project. Once the line is partially enumerated, the local optimum may be found via a simple curve fit to a quadratic polynomial. Using the local optimum determined from the partial line enumeration, a subsequent iteration begins with a gradient evaluation at this point. The SLP process continues based on optimization convergence criteria or schedule considerations.

The SLP algorithm is implemented using Matlab and uses the lingprog LP solver to determine search directions. The responses required to calculate the objective and the constraints are supplied via the SORCER Engineering ESAV Model Provider. The communication between Matlab and the Engineering ESAV Model Provider is done by instantiating the SORCER ModelClient class within a Matlab session. The ModelClient class provides a simple interface for setting design variable values, running the MDA, and obtaining the responses necessary to form the objective and constraint functions.

\section{Results}

Figure 6 shows the planforms for the baseline (approximately $1550 \mathrm{mi}$ range and $13,125 \mathrm{lb}$ half-span weight), the $1500 \mathrm{mi}$ range design (13,013 $\mathrm{lb}$ half-span weight), and the $2500 \mathrm{mi}$ range design (14,065 lb half-span weight). The optimization iteration history is shown in Figure 7 for the design variables, constraint (range), and objective (weight). Figure 8 plots the results of the ASTROS structural sizing sub-optimization performed on the three ESAV designs. The figure shows the optimal thicknesses of the skins, ribs, and spars for the three configurations.

In the first case with the $1500 \mathrm{mi}$ range constraint, the wing area and taper ratio were reduced to minimize weight. Since the baseline range was slightly greater than $1500 \mathrm{mi}$, the optimizer had some design freedom to reduce the objective function in this manner. The overall reduction in weight from the baseline was approximately $0.9 \%$. The structural sizing suboptimization was driven in this case by two of the four maneuvers: the $9.0 \mathrm{~g}$ pull-up at Mach 0.9 ; and the $7.2 \mathrm{~g}$ pull-up at Mach 1.2.

The second optimization problem solved was for the $2500 \mathrm{mi}$ range constraint. The planform of the wing in this case became long and slender with a high aspect ratio, high wing area, and low taper ratio. Since the initial design was infeasible, a considerable amount of weight was added via increases in wing area and aspect ratio to achieve the $2500 \mathrm{mi}$ range. The weight was increased approximately $7.2 \%$ from the baseline design. The structural sizing suboptimization was driven by the $9.0 \mathrm{~g}$ pull-up at Mach 0.9 maneuver.

\section{Conclusions}

The SORCER engineering software was used successfully in the design of two prototype ESAVs. The results from both optimization cases exhibited the correct trends consistent with historical aircraft design. These results provide a degree of 
validation of the implementation of the Matlab SLP code, the SORCER ESAV model, the SORCER providers, and the JavaSpaces technology.

The use of the JavaSpaces technology for parallel distributed computing proved reliable and efficient. It was a straightforward process to add computers to the SORCER grid as needed during the course of the two optimization studies. This flexibility proved valuable as the number of computers available varied from day-to-day.

Lastly, the application of a gradient-based SLP optimization technique capable of handling failed design points was demonstrated successfully. The robust nature of the SLP technique and its ability to perform gradient and line search functions in parallel made the ESAV optimization studies possible in a short period of time with minimal user intervention.

Although the SLP technique did not necessarily determine a global optimum, it did: (1) find a feasible design when started from an infeasible one; (2) improve the objective function when started from a feasible design; and (3) operate autonomously while providing the user with feedback on the optimization process. The results demonstrate the practical utility of the robust SLP approach. Furthermore, the algorithm was straightforward to implement, troubleshoot, and its successive nature (i.e., design improvements with each iteration) provided re-start capability. These qualities make it a sound approach for design optimization, especially when working with newly implemented MDAs where the univariate sweeps executed for the finite-difference sensitivities provide valuable feedback to the user.

Future work will include using design space exploration techniques and emergent optimization methods, such as genetic algorithms. Also, varying the fidelity of several of the analyses in the MDA in a variable complexity optimization process may be examined.

\section{References}

1. Integration of tools and processes for affordable vehicles, Tech. Report RTO-TR-AVT-093 AC/323(AVT-093)TP/102, NATO Research and Technology Organisation, 2006.

2. Pre-Milestone A and Early-Phase Systems Engineering: A Retrospective Review and Benefits for Future Air Force Acquisition, Committee on Pre-Milestone A and Early-Phase Systems Engineering: A Retrospective Review and Benefits for Future Air Force Acquisition, National Research Council, ISBN: 0-309-11476-4, 2008.
3. Fantini P, Balachandran LK, Guenov MD. 2008. Computational system for multi disciplinary optimization at conceptual design stage. International Journal of Simulation and Multidisciplinary Design Optimization, 2, 177-185.

4. Schumacher G, Daoud F, Petersson O, Wagner M. 2012. Multidisciplinary airframe design optimization, 28th International Congress of the Aeronautical Sciences.

5. Kolonay RM, Sobolewski M. 2011. Service ORiented Computing EnviRonment (SORCER) for large scale, distributed, dynamic fidelity aeroelastic analysis \& optimization, International Forum on Aeroelasticity and Structural Dynamics, Paris.

6. Alyanak E, Kolonay R. 2012. Efficient supersonic air vehicle structural modeling for conceptual design, 12th AIAA Aviation Technology, Integration, and Operations (ATIO) Conference and 14th AIAA/ISSM, Indianapolis, DOI: 10.2514/6.20125519

7. Neill D, Herendeen D. 1995. ASTROS Enhancements: Volume I - Astros User's Manual, Wright Laboratory WL-TR-96-3004.

8. Mason WH. 2002. Software for Aerodynamics and Aircraft Design. Retrieved 2012, from Skin Friction/Form Factor Drag estimation: http://www.dept.aoe.vt.edu/ mason/Mason_f/MRsoft. html\#SkinFriction

9. Melin T. 2000. A Vortex Lattice MATLAB Implementation for Linear Aerodynamic Wing Applications, Royal Institute of Technology, http://www.redhammer.se/tornado

10. Macinnis DV. 1994. A Simplified Cycle-Matching Transient Simulation of a Turbofan Engine, AIAA Joint Propulsion Conference, Indianapolis.

11. Newmarch J. 2006. Foundations of Jini 2 Programming, Apress, Inc., Chap. 1.

12. Freeman E, Hupfer S, Arnold K. 1999. JavaSpaces Principles, Patterns, and Practice, Addison Wesley Longman Inc., Chap. 1.

13. Argod V, Belegundu AD, Aziz A, Agrawala V, Jain R, Rajan SD. 2008. MPI-enabled shape optimization of panels subjected to air blast loading. International Journal of Simulation and Multidisciplinary Design Optimization, 2, 273-282.

14. Raymer D. 1992. Aircraft Design: A Conceptual Approach, 2nd ed., AIAA Education Series, Chap. 18.

15. Reklaitis GV, Ravindran A, Ragsdell KM. 1983. Engineering Optimization: Methods and Applications, John Wiley \& Sons, Inc., New York, Chap. 8.

16. Burton SA, Prakash C, Machnaim J. 2007. Multistage Low Pressure Turbine Airfoil Shape Optimization using the $C^{3}$ Process, 3rd AIAA Multidisciplinary Design Optimization Specialist Conference, Honolulu.

Cite this article as: Burton SA, Alyanak EJ \& Kolonay RM: Efficient supersonic air vehicle design using the Service-Oriented Computing Environment (SORCER). Int. J. Simul. Multisci. Des. Optim., 2014, 5, A25. 